\title{
Evaluating Utility of Cold-Injury Syndromic Surveillance Data in New York City
}

\author{
Kathryn Lane*1, Ramona Lall ${ }^{2}$, Katherine Wheeler ${ }^{1}$, Kazuhiko Ito ${ }^{1}$ and Thomas Matte ${ }^{1}$ \\ 'Bureau of Environmental Surveillance \& Policy, NYC Department of Health \& Mental Hygiene, New York, NY, USA; ${ }^{2}$ NYC Department \\ of Health \& Mental Hygiene - Bureau of Communicable Diseases, New York, NY, USA
}

\section{Objective}

1) Develop cold exposure-related injury syndromic case definitions 2) use historical data to compare trends among cases identified in syndromic surveillance and cases identified in NY Statewide Planning and Research Cooperative System (SPARCS) hospital discharge data to evaluate representativeness and 3) develop regression models to examine relationships with cold weather conditions, and compare relationships across case definitions and data sources.

\section{Introduction}

Cold weather exposure-related injuries range from hypothermia to less severe conditions such as frost bite, trench foot, and chilblains, which are all preventable causes of mortality and morbidity. In recent years, NYC has successfully used syndromic surveillance of heat-related ED visits to inform emergency response during heat waves. Similar timely surveillance of cold-exposure related injuries could also inform public health protection measures during severe winter weather or cold season power outages. We conducted a retrospective analysis to compare hypothermia and cold-injury patient case characteristics, as well as temporal and meteorological correlates, between syndromic surveillance data and hospital discharge data.

\section{Methods}

Using chief complaint key words, we developed syndromic case definitions for 1) hypothermia only and 2) all injury caused by environmental cold exposure. Case definitions were applied to an archive of 2008-2010 cold season (October to April) syndromic surveillance data reported from a subset of NYC emergency departments (ED SS), representing $95 \%$ of all ED visits in NYC. Relevant ICD-9 codes (991, E901.0, E901.8, E901.9, E988.3) were applied to ED discharge data (ED Dx) to detect hypothermia and cold injury cases. Age, gender, and alcohol involvement were compared using tests of proportion to determine whether characteristics of cases identified through ED SS were representative of cases identified in ED Dx data. Poisson regression models were fit to estimate the relation of daily ED SS and Dx counts with daily temperature, snow depth, and other weather conditions. Models were adjusted for month, holiday, day of week, and year to account for potential temporal confounding.

\section{Results}

Fewer hypothermia and cold injury cases were detected with ED SS than with ED Dx but the two populations did not differ significantly with respect to age and sex. From 2008-2010, there were 292 hypothermia cases with an average annual rate of 1.2 per 100,000 people, and 445 cold injury cases $(1.8$ per 100,000$)$ identified in ED SS data. Over the same time period, there were 566 hypothermia cases $(2.3$ per 100,000$)$ and 933 cold injury cases $(3.7$ per 100,000$)$ identified in ED Dx data. Daily counts of hypothermia and cold injury were correlated across data sources. In preliminary analyses using both case definitions, minimum daily temperature was associated with increases in daily ED SS and ED Dx counts. Mean daily snow depth was associated with ED SS and ED Dx cold injury cases, although not with hypothermia counts. Risk increased at lower temperatures for both case definitions.

\section{Conclusions}

There were no meaningful differences between ED SS and ED Dx weather models. Minimum temperature is associated with both case definitions. Snow depth is associated with cold injury. Daily minimum temperature and mean snow depth are potentially useful in determining timing of surveillance. Syndromic surveillance data are a timely means for monitoring hypothermia and other cold-related injuries, and could provide health departments with useful information during severe winter weather to guide prevention.

\section{Keywords}

syndromic; cold-injury; environmental surveillance

\section{Acknowledgments}

Data Analysis and Syndromic Surveillance Unit, Bureau of Communicable Diseases, NYC DOHMH

\section{*Kathryn Lane}

E-mail: klane1@health.nyc.gov 